\title{
Heart Rhythm Classification Using Short-term ECG Atrial and Ventricular Activity Analysis
}

\author{
Sasan Yazdani*, Priscille Laub, Adrian Luca, Jean-Marc Vesin \\ Applied Signal Processing Group, Swiss Federal Institute of Technology, Lausanne, Switzerland
}

\begin{abstract}
As a contribution to 2017 Physionet/CinC challenge, this work aims at the classification of different ECG heart rhythms. The importance of heart rhythm classification cannot be understated, as rhythms such as atrial fibrillation have been associated with stroke, coronary artery disease and mortality. Automatic detection of heart rhythms remains a challenging task, as they can be episodic with unpredictable characteristics. In the CinC2017 challenge, the training set contains 8528 single lead short-term ECGs (9-90s, $300 \mathrm{~Hz})$. Recordings are categorized into four classes namely, normal rhythm, atrial fibrillation, other rhythm, and noisy. Heart rhythm classification in this work is carried out by analyzing the atrial and ventricular activities present in the ECG. First, Noisy signals are classified using a Bagging meta-algorithm, trained on a set of features extracted from short- and long-term ECG trends. Then, using a novel QRS-complex cancellation technique, atrial activity is separated and used to extract several features using phase-rectified signal averaging and complexity measures. These features are then combined with heartrate variability and average-beat analysis features, to create the final feature set. The heart rhythm type is determined by a normal vs abnormal rhythm classification (Bagging meta-algorithm), followed, if needed, by an AF vs other rhythm classification (SVM classifier). The performance on the validation set led to an average F-score of 0.91 with normal, other and AF rhythm F-score of 0.95, 0.93, 0.90. On the hidden test set, our algorithm obtained an average $F$-score of 0.79 .
\end{abstract}

\section{Introduction}

The electrocardiogram (ECG), captured at body surface, is comprised of different electrical waveforms each representing depolarization or repolarization of different heart chambers. Studied for over a century, ECG can provide valuable information such as heart rate, normal/abnormal heart activities and rhythms that can determine patient health and help physicians with the appropriate medication to prescribe. Heart rhythm analysis is especially of high importance as it can help predict progression of certain arrhythmias such as atrial fibrillation (AF), for example from paroxysmal to permanent $\mathrm{AF}[1]$.

The aim in 2017 PhysioNet/CinC Challenge was the development of algorithms to classify single lead, short ECG recordings (9 -90 seconds) into normal sinus rhythm $(\mathrm{N})$, atrial fibrillation (AF), other rhythm (O), or too noisy (X). The AF class highlights the importance of this heart rhythm, with millions of people suffering form AF and projections reporting AF prevalence augmentation over the next decades [2][3]. AF is the most common type of sustained cardiac arrhythmia, characterized by a complex behavior that leads to rapid and irregular atrial activation [1][4]. Long-term AF has been associated with stroke, coronary artery disease and mortality [4].

Due to the unpredictability of characteristics of AF and other rhythms, automatic classification of heart rhythms is still problematic. Generally, AF detection methods are based on atrial and/or ventricular activity analysis. In the former, the presence of fibrillatory F-wave [5] or absence of P-waves [6] are analyzed. Other works used wavelet entropy [7] and wavelet energy [8] to detect AF. In the latter approaches, AF detection is carried out using the inter-beat intervals (RR) by techniques such as irregularity detection [9], sample entropy [10] and Shanon entropy [11]. Noise in the ECG can affect moderately to highly AF detection [12]. Therefore, it is important to have an automatic noise detection to eliminate un-processable segments to lower the heart rhythm classification error.

\section{Methods}

This study aims at the classification of not only AF vs normal heart rhythm, but also at noisy ECG detection and abnormal heart rhythm classification. The proposed approach relies on both atrial and ventricular activity analysis, based on a novel non-linear filtering technique recently proposed to extract short-term events from biomedical signals [13][14][15]. A block diagram of the proposed method is represented in Fig. 1. 


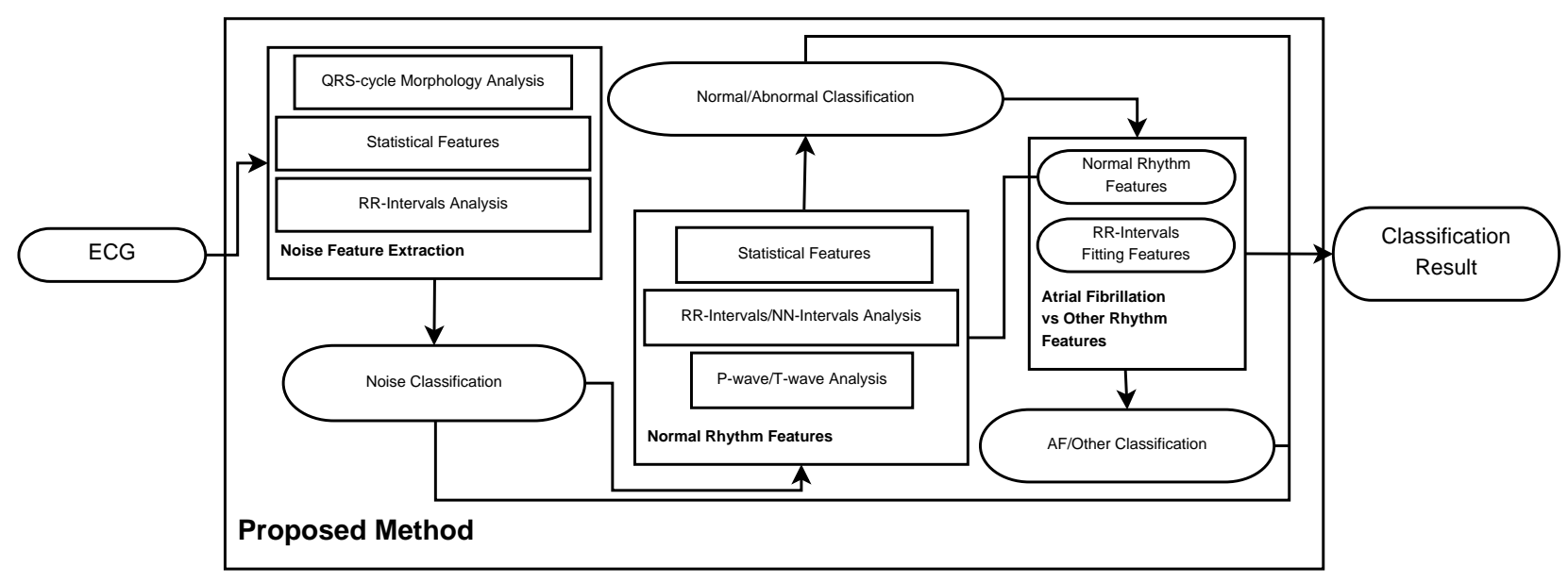

Figure 1. Block diagram of the proposed method.

\subsection{Challenge Data}

For this challenge, the data comprise ECGs collected using the AliveCor recording device, with a training set of 8,528 single-lead ECG recordings sampled at $300 \mathrm{~Hz}$ lasting from 9 -90 seconds, and a hidden test set of 3,658 ECGs of similar lengths [16]. Training recordings were manually labeled and rechecked into normal (60.4\%), AF (9.0\%), other rhythm (30\%), and noisy $(0.5 \%)$.

\subsection{The Noise Class}

The first step of the proposed approach is to determine whether the recording is of acceptable quality for heart rhythm detection. Initially, each tape is segmented into four subsegments and peak-to-peak amplitude, mean Rpeak values, root-mean-squares of iso-electric sections (as proposed in [17]), and QRS-cycle morphology correlation between heartbeats are extracted as features. Concurrently, using the non-linear filtering technique described in [13] QRS-complexes are extracted and removed from the ECG. Then, secondary statistical features such as mean, standard deviation, and median of the residual signal are calculated both on the full recording and on sliding windows. Finally, with the technique described in [15], four RR-intervals are extracted, using different parameters and thresholds, and compared to extract tertiary features for noise assessment in the ECG. It is noteworthy that over the course of this competition, due to imperfect labeling, several recording labels were modified to noisy class.

The extracted features are then combined and used to train a Bagging ensemble of classifiers with decision treebase classifier. The trained noise classifier, reached an efficient F-score of 0.85 on the validation set. However, as the data was highly unbalanced (even after label modification), and the recordings were imperfectly labeled in the training set, the assessment on the noise class is not thorough. The highly unbalanced noise class might be a reason why the noise F-score was removed from the final scoring system of the challenge.

\subsection{Normal vs. Abnormal Classification}

Once the ECG is determined to be of acceptable quality, normal sinus rhythm is studied. First R-waves are extracted (see [13]), and the RR-intervals time series is extracted. RR-intervals are then further processed by two sets of thresholds on time and amplitude to extract two normal-to-normal intervals (NN-intervals). Subsequently, using dynamic time warping [18], the distance between the original and the two extracted NN intervals are used as features. Furthermore, the distances between RR- and NNintervals are computed by scrolling the shorter signal on top of the longer one and selecting the minimum distance. The difference signal is then used to extract statistical features such as mean, standard deviation, and peak-to-peak amplitude difference. Then, these features are added to the features previously extracted.

At the same time, for each heartbeat, using the technique described in [19], the QRS-complexes, P-waves and $\mathrm{T}$-waves are extracted. Features such mean and standard deviation of correlation coefficients of QRS-complexes, Pwaves, and T-waves are extracted and combined with amplitude and duration features in order to complement the RR-intervals driven features.

Similarly to the noise class, the extracted features are then combined and used to train a Bagging ensemble of classifiers with decision tree base classifier. The Normal classifier reached an F-score of 0.95 on the validation set, while the F-score on the hidden test set was 0.90. It should be emphasized that the data was highly unbalanced towards the normal class. It is also noteworthy that after veri- 

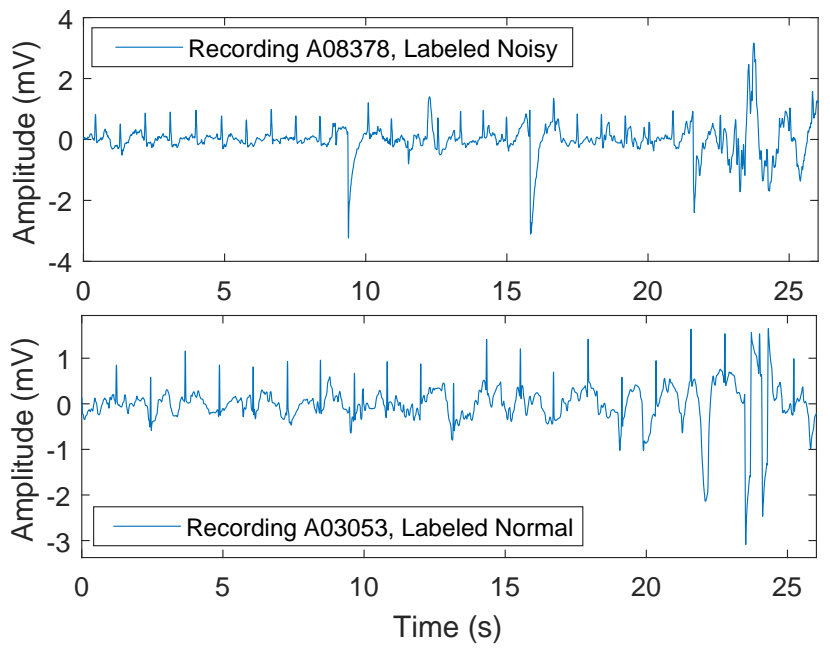

Figure 2. Imperfect labeling of recordings in the dataset.

fying the misclassification of normal to abnormal class, observations showed that misclassification were mostly due to impulsive or noisy ECG segments. This behavior is of course expected as noisy segments have a direct influence on the features. It seems that there was not a proper consensus in noisy tape labeling, as some tapes were considered "normal" while other tapes with similar or worse behavior were labeled as "noisy." An example is given in Fig. 2.

\subsection{Atrial Fibrillation vs. Other Rhythms Classification}

The final stage of the proposed method is to classify the abnormal rhythms detected in subsection 2.3. This stage uses features similar to the ones described in subsection 2.3. However, our observations showed that heart rate variability indexes, especially non-linear ones such as sample entropy, approximate entropy, and multi-scale entropy, could help improve the AF vs other-rhythm classification performance. Furthermore, polinomials of orders 5 and 15 were fitted on the different RR-intervals, the original as well as the cleaned intervals [see subsection 2.3], and features based on element-wise distances between the fitted and the original signals were used to scrutinize the intervals for impulsive changes such as premature beats, bigeminy, and trigeminy.

Similarly to previous subsections, the extracted features were finally combined to train a Bagging ensemble of learners with decision tree classifiers. The $\mathrm{AF}$ and otherrhythm ensemble of classifiers respectively obtained Fscores of 0.93 and 0.90 on the validation set, while the F-scores on the hidden test set were 0.77 and 0.69. Based on our observations on the training and validation sets, we believe the difference between the validation set and the
Table 1. Performance details of the proposed scheme.

\begin{tabular}{|c|c|c|c|}
\hline & Unofficial Phase & \multicolumn{2}{|c|}{ Official Phase } \\
\hline & Test set & Validation & Test set \\
\hline Normal & 0.82 & 0.95 & N/A \\
\hline $\mathrm{AF}$ & 0.72 & 0.93 & N/A \\
\hline Other & 0.68 & 0.90 & N/A \\
\hline Noisy & 0.57 & $0.85 *$ & N/A \\
\hline Final Score & 0.70 & 0.92 & 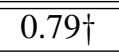 \\
\hline
\end{tabular}

hidden test set are due miss classifications on the normal class. As the normal class constitutes more than $60 \%$ of the training set (and probably the hidden test set), miss classifications of 100 tapes, for instance, can barely effect the total F-score of the normal class while it has drastic effects on other rhythms.

\section{Results and Discussion}

The Physionet/CinC2017 challenge was divided into an unofficial and an official phase. For the unofficial phase the final score was calculated as the average of F-scores on all four classes, namely normal, atrial fibrillation, other rhythm and noisy. As the number of noisy recordings was considerably smaller than that of other classes, in order to have a fairer evaluation method, the F-score of the noisy class was removed and the official phase final score was computed as the average of F-scores on the normal, atrial fibrillation and other rhythms [16].

Table 1 shows the results obtained for the unofficial and official phases of this challenge. Although during the unofficial phase, the labels of some recordings were altered to clean imperfect labeling, the number of noisy recordings was still too small to consider an F-score in the final scoring function of the challenge. Final performance on the training set led to an F-score of 0.87 , and with the validation set score of 0.92 . It is worth mentioning that the recordings provided in the validation set do not have the same class distribution as the training set. Of course, as the distributions were highly unbalanced, the F-score is more representative of performance compared to classification accuracy and therefore, is a suitable choice for the scoring function.

In conclusion, this paper presents a framework to identify short-term ECG recording rhythms. Features based on heart-rate variability, RR-intervals, and atrial/ventricular ECG morphologies were extracted and used to train a serialized ensemble of classifiers to classify the ECG rhythm into normal, atrial fibrillation, other abnormal rhythms, or too noisy to classify. 


\section{Acknowledgements}

This study was performed in the framework of the NanoTera initiative supported by the Swiss National Science Foundation (SNSF).

\section{References}

[1] Fuster V, Rydén LE, Cannom DS, Crijns HJ, Curtis AB, Ellenbogen KA, Halperin JL, Le Heuzey JY, Kay GN, Lowe JE, et al. Acc/aha/esc 2006 guidelines for the management of patients with atrial fibrillation: full text: a report of the american college of cardiology/american heart association task force on practice guidelines and the european society of cardiology committee for practice guidelines (writing committee to revise the 2001 guidelines for the management of patients with atrial fibrillation) developed in collaboration with the european heart rhythm association and the heart rhythm society. Europace 2006;8(9):651-745.

[2] Naccarelli GV, Varker H, Lin J, Schulman KL. Increasing Prevalence of Atrial Fibrillation and Flutter in the United States. The American Journal of Cardiology December 2009;104(11):1534-1539. ISSN 0002-9149.

[3] Savelieva I, Camm J. Update on Atrial Fibrillation: Part I. Clin Cardiol February 2008;31(2):55-62. ISSN 1932-8737.

[4] G.Y.H. Lip L. Fauchier SFIVGANCGSNTPMRHTDL. Atrial fibrillation. Nature Reviews Disease Primers March 2016; vol 2:2. ISSN 2056-676X.

[5] Du X, Rao N, Qian M, Liu D, Li J, Feng W, Yin L, Chen X. A Novel Method for Real-Time Atrial Fibrillation Detection in Electrocardiograms Using Multiple Parameters. Annals of Noninvasive Electrocardiology May 2014;19(3):217-225. ISSN 1542-474X.

[6] Ladavich S, Ghoraani B. Rate-independent detection of atrial fibrillation by statistical modeling of atrial activity. Biomedical Signal Processing and Control April 2015; 18:274-281. ISSN 1746-8094.

[7] Ródenas J, García M, Alcaraz R, Rieta JJ. Wavelet entropy automatically detects episodes of atrial fibrillation from single-lead electrocardiograms. Entropy 2015;17(9):6179_ 6199.

[8] García M, Ródenas J, Alcaraz R, Rieta JJ. Application of the relative wavelet energy to heart rate independent detection of atrial fibrillation. computer methods and programs in biomedicine 2016;131:157-168.

[9] Petrenas A, Marozas V, Sörnmo L. Low-complexity detection of atrial fibrillation in continuous long-term monitor- ing. Computers in Biology and Medicine October 2015; 65:184-191. ISSN 0010-4825.

[10] Alcaraz R, Abásolo D, Hornero R, Rieta JJ. Optimal parameters study for sample entropy-based atrial fibrillation organization analysis. Computer methods and programs in biomedicine 2010;99(1):124-132.

[11] Zhou X, Ding H, Ung B, Pickwell-MacPherson E, Zhang Y. Automatic online detection of atrial fibrillation based on symbolic dynamics and shannon entropy. Biomedical engineering online 2014;13(1): 18 .

[12] Oster J, Clifford GD. Impact of the presence of noise on RR interval-based atrial fibrillation detection. Journal of Electrocardiology November 2015;48(6):947-951. ISSN 0022 0736.

[13] Yazdani S, Fallet S, Vesin JM. A Novel Short-term Event Extraction Algorithm for Biomedical Signals. IEEE Transactions on Biomedical Engineering 2017;PP(99):1-1. ISSN 0018-9294.

[14] Vesin JM, Yazdani S, Mirmohamadsadeghi L, Bourdillon N. Extraction and analysis of short-time excursions in RRinterval time series. In 2016 Computing in Cardiology Conference (CinC). September 2016; 721-724.

[15] Yazdani S, Vesin JM. A novel preprocessing tool to enhance ECG R-wave extraction. In 2016 Computing in Cardiology Conference (CinC). September 2016; 633-636.

[16] Clifford G, Liu C, Moody B, Lehman L, Silva I, Li Q, Johnson A, Mark R. Af classification from a short single lead ecg recording: The physionet computing in cardiology challenge 2017. In 2017 Computing in Cardiology Conference (CinC). September 2017; to be published.

[17] Clifford GD, Behar J, Li Q, Rezek I. Signal quality indices and data fusion for determining clinical acceptability of electrocardiograms. Physiol Meas 2012;33(9):1419. ISSN 0967-3334.

[18] Sakoe H, Chiba S. Dynamic programming algorithm optimization for spoken word recognition. IEEE transactions on acoustics speech and signal processing 1978;26(1):4349.

[19] Lemay M, Vesin JM, Van Oosterom A, Jacquemet V, Kappenberger L. Cancellation of ventricular activity in the ecg: evaluation of novel and existing methods. IEEE Transactions on Biomedical Engineering 2007;54(3):542-546.

Address for correspondence:

Sasan Yazdani

EPFL SCI STI JMV - ELE 121 - Station 11

CH-1015 Lausanne - Switzerland.

E-mail address: sasan.yazdani@epfl.ch 